\title{
Routes of Infections, Inflammation, Immunity, Immunity Response and Inflammatory Injury: Elucidation of a Biological Fight
}

\section{Rajiv Kumar}

NIET, National Institute of Medical Science, India.

Corresponding Author: Rajiv Kumar, NIET, National Institute of Medical Science, India.

Received Date: November 13, 2021; Accepted Date: December 31, 2021; Published Date: January 07, 2022

Citation: Rajiv Kumar. (2022) Anaphylaxis Induced by Beer. J. Immunology and Inflammation Diseases Therapy. 5(1); Doi:10.31579/2637$8876 / 028$

Copyright: () 2022 Rajiv Kumar. This is an open-access article distributed under the terms of The Creative Commons Attribution License, which permits unrestricted use, distribution, and reproduction in any medium, provided the original author and source are credited.

\begin{abstract}
:
Infections, inflammation, immunity, and inflammatory injury are different segments of biological events and link up altogether. Route of infection has no similarity with the cellular signaling pathway of inflammation, even though when inflammation is induced by infection. The organism responds toward infection that is initiated by the pathogen via inflammation, which is a natural way of defense initiated by innate immunity as a safeguard
\end{abstract}

Keywords : Infections; inflammation; immunity; and inflammatory

\section{Introduction}

Infections, inflammation, immunity, and inflammatory injury are different segments of biological events and link up altogether. Route of infection has no similarity with the cellular signaling pathway of inflammation, even though when inflammation is induced by infection. The organism responds toward infection that is initiated by the pathogen via inflammation, which is a natural way of defense initiated by innate immunity as a safeguard. [1] Disorders/diseases can instantaneously come about. The progression or exacerbation of the disease openly or incidentally may disturb or support the other ailment. Mucormycosis, a black fungus, group of fungi known as mucormycetes, a dangerous fungal infection is entering in patients who are being affected by a coronavirus (Figure 1).

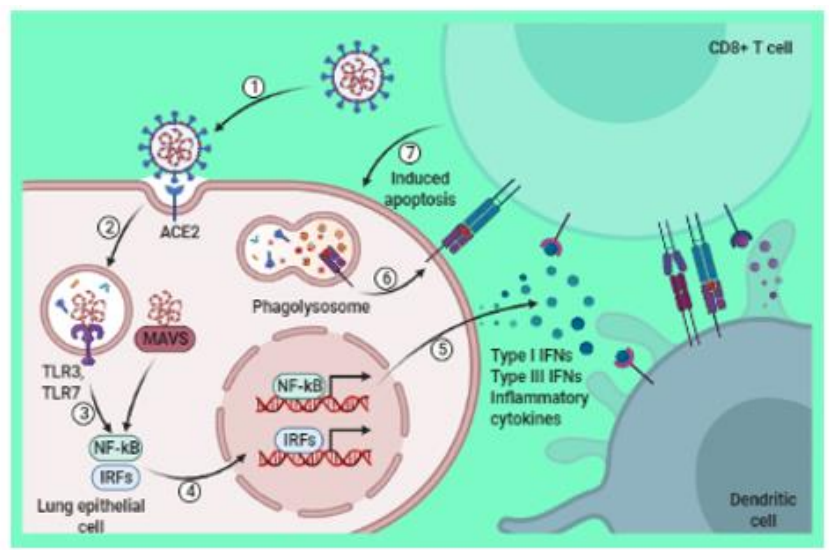

Figure 1: An elucidation of acute immune responses to coronaviruses. 
Microorganisms (viruses, prions, bacteria, viroids, parasites, and fungi) and their components (antigens) trigger inflammation. The proinflammatory immune mediators, i.e., cytokines, chemokines, and Creactive protein produced by leukocytes, endothelial cells, and hepatocytes naturally counter the bacteria/virulence infection.

Meanwhile, soluble antigen and specific antibody collectively form immune complexes that intensify inflammation at the location of the deposition. Locally produced IL-1, IL-6, TNF- and PGE2 proinflammatory mediators initiate endothelial dysfunction. Severe inflammatory conditions originate in different diseases, including osteomyelitis, rheumatoid arthritis, sarcoidosis, inflammatory bowel disease, and fever of unknown cause.[2] When any bacteria, virus, or injurious agent triggers the release of histamine, an inflammatory secreted from mast cells, damaged lining cells of blood vessels, and platelets express the initiation of the disease phase.[3]

During acute inflammation, white blood cells destroy the bacteria and eradicate tissue debris. Granulation tissue migrates and matures into stable cells for dealing with wound healing. Chronic inflammation originates at the time of chronic diseases, i.e., osteomyelitis, tuberculosis, bowel inflammation, but simultaneously the process of demolition and repair also takes place at the affected area.[4] The interaction between host and microorganism was elaborated during the beginning and treatment of allergies, and autoimmune diseases, including inflammation in mucus, membranes, and tuberculosis. Resistance to antibiotics detected against lactobacilli, staphylococcus, mycobacteria, enterococcus, and chlamydia, borrelia. The epithelial barrier transmits signals to epithelial cells and confirmed bacterial invasion. These biological events encourage cells to produce inflammatory responses to inhibit microbial infection. Various routes were followed by the epithelial cells to identify microorganisms and fretful constituents. Urgent needs arise to explain these mechanisms that will expose the innate immune responses. This elucidation decodes the proceeding of immunology of mucosal surfaces and microbial pathogenesis. Interleukin 6 (IL-6) govern the route of initiation of response toward infections and tissue injuries via the stimulation of acute-phase responses, immune reactions, and haematopoiesis.[5]Transcriptional and post-transcriptional mechanisms control a few biological events. If any dysregulation occurs in the synthetic route of IL-6, it will induce a pathological effect on chronic inflammation and autoimmunity.[6 ]Epidemiologic analysis proved interconnected routes of infectious agents, chronic inflammatory disorders, and diseases.[7] Therefore, a better elucidation of microbial pattern-recognition receptors and innate immune signaling pathways exposes the association of microbial infection and chronic disease. Innate response recruits the secretion of cytokines (TNF- $\alpha$, IL-1 $\beta$, and IL-6) after recognition of the microbial agents. Among them, IL-1 $\beta$ and IL- 6 direct inflammatory cell migration and osteoclastogenesis.

TNF- $\alpha$ is stimulus cell migration and plays a part in tissue destruction as well as take part in creating IL-1 $\beta$ and IL-6. It is also associated with extracellular matrix degradation. Chemokine control migration of phagocytic cell towards the location of infection. Chemotactic factors forced leucocytes for migration toward the location of infection. The endothelial, leucocytes, epithelial, and stromal cells shaped chemokine that performed cell trafficking and transformed later on into homeostatic. Chemokine is executed as a messenger and plays a key role in angiogenesis, host defense, cell proliferation, apoptosis, and tumor metastasis. Chemokine stimulus several features of leucocytes and lymphocytes. The initiation of abnormalities in the epithelial barrier is a key aspect of pathogenesis; the presence of microbial toxins, genetic predisposition of the host, and environmental insults worsens it. The failures of defense regularization inhibit leukocyte activation and the resulting cascades initiate chronic inflammation and its consequences.[8] Interpreting the impact of microbial agents on cellular events and observations of routes promotes diverse conditions that induce diseases, including Crohn's disease, diabetes, cancer, psoriasis, coronavirus, and atherosclerosis, will expose the looked-for feature.[9] In many infected diseases, the fundamental problem is how to control thrombosis. In patients with diabetes, the dose of antibiotics and antifungal medicines should be prescribed accurately.

Management of diabetes and discontinuing immunomodulation can also suggest.[10] Proper regularization of micronutrient homeostasis is very helpful for a healthy immune structure. Zinc deficiency interrupts the natural regulation of micronutrient homeostasis, proinflammatory response, and initiates damage to host tissue. Zinc influences transcription factors, nuclear factor kappa $\mathrm{B}(\mathrm{NF}-\kappa \mathrm{B})$, controls oxidative stress and plays a key role in the regularization of inflammatory cytokines.[11] The physical, chemical, or immunological agents can initiate injury and inflammation is a biological event as a tissue reaction.[12] However, when microbes caused an injury that can lead to an infection. The inflammatory response is a complex network of immunologic and histochemical events, including the release of chemical mediators (histamine, serotonin, multiple cytokines, chemokines, platelet-activating factor, nitric oxide, bradykinin, and arachidonic acid metabolites) and vasodilation with higher vascular permeability.[13] Intra-abdominal abscesses, infections of prosthetic devices, endocarditis, and osteomyelitis are well-rooted infections, and it is not as easy to detect. Modern scientific techniques and methods i.e. nuclear medicine techniques for infection/inflammation imaging, $67 \mathrm{Ga}$-citrate, $99 \mathrm{mTc}$ or 111In-labeled leukocytes, $99 \mathrm{mTc}$ or $111 \mathrm{In}$-labeled human immunoglobulin (HIG), 99mTc labeled granulocyte antibodies (AGAbs), Radiolabeled liposomes, 99mTc-nanocolloids, Avidin or streptavidin/indium-111 labeled biotin, Radiolabeled cytokines, and chemokines, Radiolabeled chemotactic peptides, 18Ffluorodeoxyglucose (18F-FDG), Properties of an ideal infection imaging agent, targeting the microorganism approaches, radiolabeled antibiotics, radiolabeled antibacterial agents, fluoroquinolones, cephalosporins, radiolabeled antitubercular agents, radiolabeled antifungal agents, radiolabeled FIAUs, radiolabeled antimicrobial peptides have been developed for an accurate diagnosis and therapeutic for the elucidation of deep-rooted infections.[14]

Herein, the elucidation of the routes of the pathophysiology of inflammation after injuries and infection is necessary because these findings can be implemented in treating diseases. Inflammation disturbs cerebral blood flow (hypo- and hyper perfusion), initiate cerebral metabolic dysfunction, damage cerebrovascular autoregulation, and triggers inadequate cerebral oxygenation. There inflammatory induced several irregularities, including excitotoxic cell damage, internal injuries, apoptotic and necrotic cell death. Authors believed that a better understanding of the multidimensional cascade of injury and infection offer different options that help single out a novel therapeutic option.[15] Few examples, the internal injuries and infection of different organs such as the heart, brain, liver, kidney, and intestine initiate life-threatening diseases including cardiovascular diseases, brain injuries, cancer, diabetes, arthritis, and joint diseases, allergies, chronic obstructive pulmonary disease, Alzheimer's disease, chronic kidney disease, and inflammatory bowel disease. Chronic inflammation also induced various kinds of diseases that cause premature mortality and morbidity, including cardiovascular diseases, cancer, diabetes mellitus, asthma-chronic obstructive pulmonary disease (Figure 2), obesity, metabolic syndrome, inflammatory bowel disease, rheumatoid arthritis, multiple sclerosis, osteoporosis, and neurological diseases via dysregulation of various signaling pathways such as nuclear factor kappa-B $(\mathrm{NF}-\kappa \mathrm{B})$, signal transducer, activator of transcription-3, etc. These inflammation-related diseases are among the major causes of mortality and morbidity worldwide and increasing day by day. 


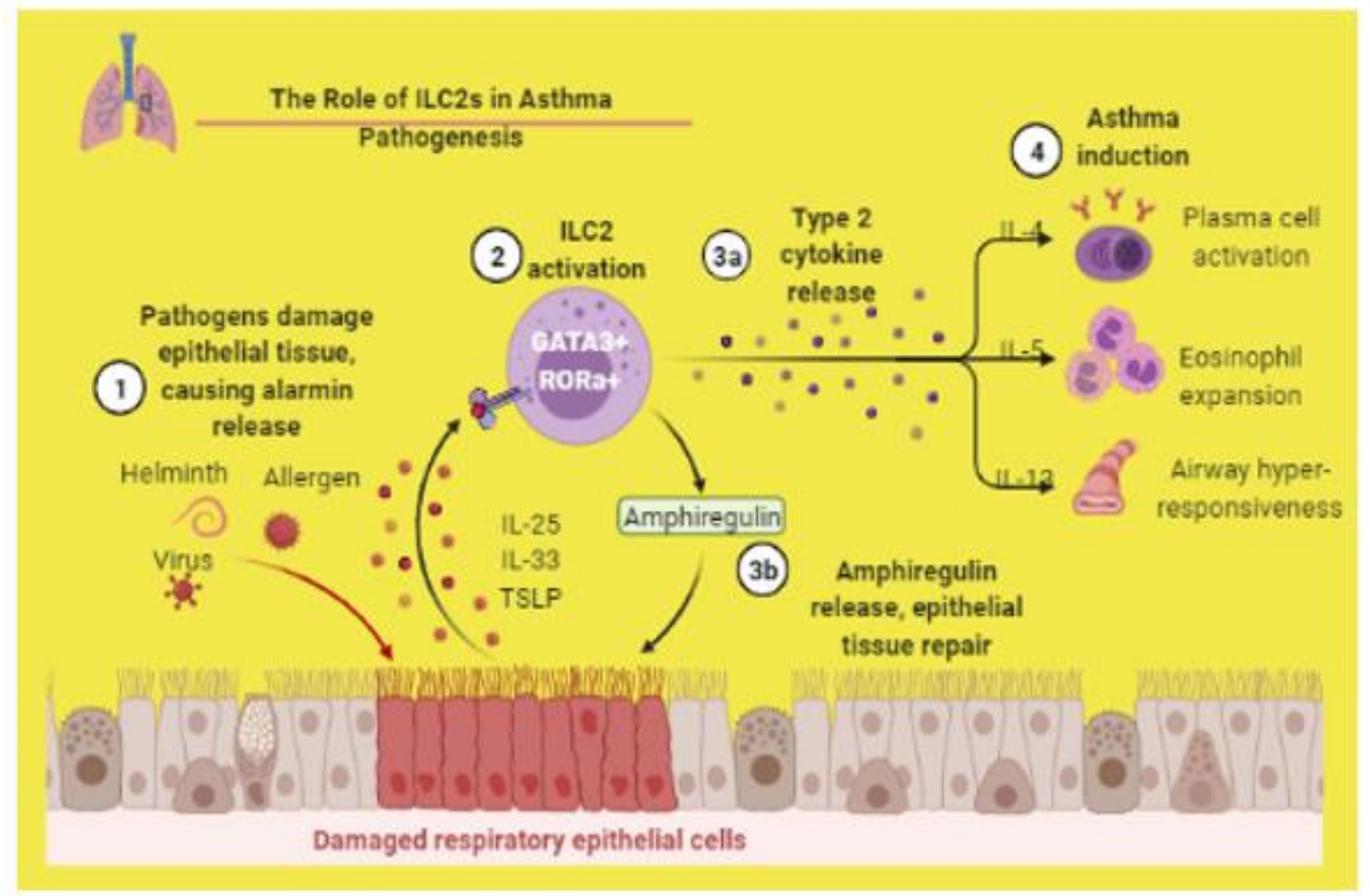

Figure 2: An elucidation of the role of ilc2s in asthma pathogenesis.

Therefore, the current article highlights inflammation and the underlying mechanisms by refereeing the physiological causes. Herein, nanotherapeutics and regenerative nanomedicines for inflammatory diseases and infection are advanced form of therapeutics that can govern cellular processes and triggered the routes of regeneration and initiate the route of healing of the damaged tissues, muscles, injuries, wounds including inflammation of various diseases and promote regeneration and cure infections noteworthy.[16] In the end, author believe that a proper elucidation of routes of infections, inflammation, immunity, immunity response and inflammatory injury will be a key approach to explore biological fight between immune defence and infecting agents. Outputs of these research interpretation and analysis will be definitely successful in the identification of the therapeutic targets.

\section{Acknowledgments}

Author (Rajiv Kumar) gratefully acknowledges his younger brother Bitto for motivation. The author acknowledges bio render for providing the facility to illustrate the diagrams (Fig. 1 and 2) and again acknowledges the same.

\section{Availability of data and materials}

Wherever necessary, relevant citations are included in the reference section.

\section{Competing interests}

The author has declared that no competing interest exists.

\section{References}

1. Mitchell G, Isberg RR. Innate Immunity to Intracellular Pathogens: Balancing Microbial Elimination and Inflammation. Cell Host Microbe. 2017; 22: 166-75.
2. Kempuraj D, Thangavel R, Selvakumar GP, et al. Brain and peripheral atypical inflammatory mediators potentiate neuroinflammation and neurodegeneration. Front. Cell. Neurosci. 2017; 11. DOI:10.3389/fncel.2017.00216.

3. Kumar R, Chhikara BS, Gulia K, Chhillar M. Cleaning the molecular machinery of cellsviaproteostasis, proteolysis and endocytosis selectively, effectively, and precisely: intracellular self-defense and cellular perturbations. Mol. Omi. 2021; 17: 11-28.

4. Bafadhel M, McCormick M, Saha S, et al. Profiling of sputum inflammatory mediators in asthma and chronic obstructive pulmonary disease. Respiration 2012 ; 83: $36-$ 44.

5. Rojas M, Restrepo-Jiménez P, Monsalve DM, et al. Molecular mimicry and autoimmunity. J. Autoimmun. 2018; 95: 100-23.

6. Salle V. Coronavirus-induced autoimmunity. Clin. Immunol. 2021; 226. DOI:10.1016/j.clim.2021.108694.

7. Morita H, Saito H, Matsumoto K, Nakae S. Regulatory roles of mast cells in immune responses. Semin. Immunopathol. 2016; 38: 623-9.

8. Abdulkhaleq LA, Assi MA, Abdullah R, Zamri-Saad M, Taufiq-Yap YH, Hezmee MNM. The crucial roles of inflammatory mediators in inflammation: A review. Vet. World. 2018; 11: 627-35.

9. Liu Y, Zhang HG. Vigilance on New-Onset Atherosclerosis Following SARS-CoV-2 Infection. Front. Med. 2021; 7. DOI:10.3389/fmed.2020.629413.

10. Walter JE, Ayala IA, Milojevic D. Autoimmunity as a continuum in primary immunodeficiency. Curr. Opin. Pediatr. 2019; 31: 851-62. 
11. Kany S, Vollrath JT, Relja B. Cytokines in inflammatory disease. Int. J. Mol. Sci. 2019; 20. DOI:10.3390/ijms20236008.

12. Angelousi A, Chatzellis E, Kaltsas G. New Molecular, Biological, and Immunological Agents Inducing Hypophysitis. Neuroendocrinology 2017; 106: 89-100.

13. Wilkes G. Targeted Therapy: Attacking Cancer with Molecular and Immunological Targeted Agents. AsiaPacific J. Oncol. Nurs. 2018; 5: 137-55.

14. Arai A. Chronic active epstein-barr virus infection: The elucidation of the pathophysiology and the development of therapeutic methods. Microorganisms 2021; 9. DOI:10.3390/microorganisms9010180.

15. Werner C, Engelhard K. Pathophysiology of traumatic brain injury Biomechanical and neuropathological classification of injury. Br J Anaesth 2007; 99.

16. Su Y, Gao J, Kaur P, Wang Z. Neutrophils and macrophages as targets for development of nanotherapeutics in inflammatory diseases. Pharmaceutics. 2020; 12: 1-24.
This work is licensed under Creative Commons Attribution 4.0 License

To Submit Your Article Click Here: $\quad$ Submit Manuscript

DOI: $10.31579 / 2637-8876 / 028$
Ready to submit your research? Choose Auctores and benefit from:

$>$ fast, convenient online submission

$>$ rigorous peer review by experienced research in your field

$>$ rapid publication on acceptance

$>$ authors retain copyrights

$>$ unique DOI for all articles

$>$ immediate, unrestricted online access

At Auctores, research is always in progress.

Learn more https://auctoresonline.org/journals/immunology-andinflammation-diseases-therapy- 